PALABRAS CLAVE: dialecto, variedades lingüísticas, doblaje, picardo, ch'ti

\title{
E1 dialecto como reto de doblaje: opciones \\ y obstáculos de la traslación de Bienvenue chez les Ch'tis
}

URSULA REUTNER

Universität Passau
Dialect as a challenge for dubbing: options and obstacles in the translation of Bienvenue chez les Ch'tis

Dubbing a film in which dialect plays a central role not only in the language of some of its characters but also as a topic of discussion and a trigger of comic misunderstandings, is a challenge for any translator. The task of transferring the successful French film Bienvenue chez les Ch'tis (Welcome to the Sticks) with its extensive use of Picard into Spanish is a case in point. In its analysis of Bienvenidos al Norte, this article discusses several solutions to the problem, rejecting traditional options such as translating dialect into standard Spanish or into a Spanish dialect like Andalusian. The creation of an artificial Spanish is put forward as the best option and evaluated in phonological and lexical terms.

KEY WORDS: dialect, language variation, dubbing, Picard, ch'ti 


\section{INTRODUCCIÓN}

La variación lingüística representa uno de los grandes retos de la traducción. Captar correctamente las connotaciones de una variedad en la lengua origen y reconstruirlas de manera adecuada en una lengua meta representa un arte. No sorprende, por lo tanto, el sinnúmero de libros y artículos que se dedican al desafío dialectal para la traducción. Recordemos sólo las reflexiones sobre la reproducción del multilingüismo en el drama shakespeareano Henry $V$ (Delabastita 2002/2005), la discusión de cómo traducir los idiolectos en la tira cómica de Astérix (Fernández/Gaspin I99I: 97s.) o en la serie televisiva The Simpsons (Fusari 2007, Muñoz Gil 2009), y el debate sobre diferentes opciones para recrear el siciliano de la novela policíaca de Camilleri (Kahn 2004, Vittoz 2004, Muñiz Muñiz 2004).

En la traducción audiovisual, la reproducción de la variación lingüística es aún más delicada, siendo las posibilidades de solución limitadas: perífrasis y explicaciones adjuntas usualmente no pueden ser consideradas ante la necesidad de conseguir un cierto ajuste labial; notas a pie de página, glosas o un glosario final no son factibles, sino en forma de subtítulos. A estas restricciones se añade la tradición del doblaje para evitar formas marcadas que ha creado una tendencia al «español neutro» o, por lo menos - siendo controvertida la cuestión de la neutralidad (cf. Ramírez Luengo 20II, Pajares Jiménez 20II) - , hacia el mínimo denominador compartido de las variedades diatópicas del castellano en forma del español común, llamado también general, global, internacional, o panhispánico.

Si el español neutro ya es criticado por su artificialidad para el doblaje de textos unilingües, resulta completamente inadecuado para películas multilingües, cada vez más frecuentes en los últimos decenios (cf. Heiss 2004).

Pensemos sólo en los trozos de seudoitaliano en la película $A$ Fish Called Wanda (Charles Chrichton 1988), de alemán en La vita è bella (Roberto Benigni 1997) o de francés en French Kiss (Lawrence Kasdan 1997).

La situación se torna aún más compleja si la segunda lengua no es - como en los ejemplos citados- una lengua extranjera con connotaciones si no idénticas, por lo menos, parecidas en diferentes países, sino una lengua bien arraigada en el país origen que se encuentra, por lo tanto, en una situación de bilingüismo o de diglosia con la primera: sea un idioma como el catalán, de importancia central en El amante bilingüe (Vicente Arranda 1997), sea un dialecto como el picardo, factor constituyente en Bienvenue chez les Ch'tis (Boon 2008). En ambos casos se trata - siguiendo la terminología de Corrius/Zabalbaescoa (20II) - de una tercera lengua, que se añade a la primera del texto origen y a la segunda del texto meta.

Dos aspectos explican el creciente desafío en estos casos: primero, dos lenguas coexistentes en un mismo país se influencian de manera que no se puede establecer una dicotomía, sino que nos enfrentamos con todo un continuum entre los dos polos. En Bienvenue chez les Ch'tis, por ejemplo, nos encontramos con personas (sobre todo personas ancianas como la señora Bailleul y, aún más, el señor Vasseur) que utilizan un fuerte dialecto y otras que hablan de un modo más cercano al estándar: la joven Annabelle es capaz de jugar con toda una variedad de registros, lo que le permite servir como intérprete entre el jefe inicialmente no picardo-hablante $y$ sus colegas ch'tis (cf. ejemplo $\mathrm{n}^{\circ}$ II en este artículo). Delimitar una frontera clara entre dos lenguas coexistentes no es siempre fácil (cf. la figura clarificadora en Zabalbeascoa, 20izb: 325), pero, a veces, de central importancia para el traductor. 
Además, una lengua arraigada en un país no está desprovista de connotaciones culturalmente específicas, que - siendo específicasconstituyen un reto especial para la traducción. Como particularidad cultural podría encajar en lo que se discute bajo el nombre de «cultural features» (Nida 196r), «culturemes» (Vermeer 1983: 8), «Kulturem» (Oksaar 1988: 26s.), «cultural words» (Newmark I988: 94-I03), «culturespecific ítems» (Franco Aixelá 1996: 52), «marcadores culturales específicos» (Herrero 2000: 3II), «culture markers» (Nord 1994) o «culturespecific phenomena» (Nord 1997) - aunque estos conceptos están en su mayoría centrados en el léxico y se refieren menos a la forma de las palabras que a su contenido.

Que la única solución para la traducción de dialectos sea la lengua estándar es un lugar común (cf. Hesse-Quack 1967: 196, Herbst 1994: I27). Esta nivelación es, en todo caso, la solución más difundida y menos laboriosa (cf. por ejemplo Baker 1993: 243ss., Agost 1999: I29, Bravo 2003: 243). La recreación de los valores dialectales del original con los medios de la lengua meta, en cambio, conlleva muchos obstáculos. No obstante, al no buscar un equivalente para el dialecto de la lengua origen, en algunos casos se pierde algo más que la información sobre la procedencia del hablante, la cual normalmente se puede deducir también de otros elementos, como el tipo de vida o el entorno. El dialecto puede representar una parte constitutiva de la acción, es decir, hacerse imprescindible para la comprensión adecuada de la historia. Esto ocurre cuando las diferencias sociales y regionales entre algunos personajes son especialmente relevantes y, sobre todo, cuando la comicidad verbal se basa en el dialecto o el dialecto en sí se convierte en tema de conversación.

No cabe duda de que una renuncia completa a recrear el dialecto conduce a una carencia de detalles importantes. Por ello, este artículo propone contemplar diferentes estrategias para resolver el problema: la verbalización directa (3.I), la sustitución del dialecto en el nivel paralingǘstico (3.2), el empleo de una variedad de la lengua meta (3.3) y la creación de un español artificial (3.4). En todos los casos se analizarán el potencial y los obstáculos de estas opciones tomando el ejemplo de Bienvenidos al Norte (Boon 2009), la versión doblada al español de la película francesa Bienvenue chez les Ch'tis (Boon 2008). Esta se presta particularmente bien para el debate de nuestro tema por dos razones: primero, porque el dialecto picardo en su variedad, el ch'ti, tiene un papel central en la película; y segundo, la opción elegida en el doblaje de adaptar algunas de sus características al español es, sin duda, insólita y digna de interés.

\section{FUNCIONES DEL DIALECTO EN BIENVENUE CHEZ LES CH'TIS}

La película cuenta la historia de un empleado de correos del sur de Francia, Philippe Abrams, que es trasladado a la región de Norte-Paso de Calais como medida disciplinaria. En el sur circulan prejuicios terribles sobre esta región: se dice que el clima es inhóspito y gélido, que sus habitantes son personas simples, groseras, incivilizadas, algo atrasadas y alcohólicas, y que se comunican en un lenguaje rudimentario.

Normalmente es el francés meridional el que, en la tradición cómica y filmográfica francesa, se emplea para despertar la connotación de mentalidad simple. En cambio, en esta película solo algunos de los personajes secundarios hablan francés meridional, mientras que es el picardo el que desempeña su papel tradicional - o mejor dicho, un picardo fingido: en línea con el concepto de «oralidad prefabricada» (cf. por ejemplo Chaume 2004: I67s., 2012: 82s), 
constatamos una «dialectalidad prefabricada» en Bienvenue chez les Ch'tis. Esa no reproduce fielmente el dialecto, sino que hace una selección de particularidades picardas de diferentes zonas para transmitir cierta autenticidad sin perder comprensibilidad para el público general. Los estereotipos franceses acerca del uso del dialecto en general y del picardo en particular permiten la identificación de los hablantes del ch'ti fílmico como personas simples y contribuyen a la imagen negativa que se construye del norte en el inicio de la película.

Una importante función del dialecto es, por tanto, reflejar de manera sintetizada la complejidad de la realidad no verbal. Por eso, resulta imposible omitir una reproducción de las características picardas en el doblaje, ya que el efecto de este tipo de películas radica, sobre todo, en las connotaciones estéticas y socioculturales que evoca el dialecto en el espectador. El uso del dialecto permite clasificar a los hablantes tanto de manera regional como, en parte, también social y, además, proporciona información sobre la relación entre hablante y oyente (función icónica o simbólica). A esto hay que añadir la función del dialecto como aspecto cómico de la película: muchas situaciones graciosas tienen su origen en malentendidos lingüísticos, que se producen por el uso del dialecto y que serían difíciles de reproducir en un registro estándar (función cómica). Al ser la recreación del humor imprescindible — según Díaz Cintas (200I: I2I) a menudo más importante que la proximidad a la semántica del original- se intenta reproducirla en Bienvenidos al Norte con diferentes propuestas, que son, en parte, originales y, en parte, poco convincentes, pero que presuponen, en su mayoría, una reproducción general del dialecto (cf. Reutner en prensa). Una última función que cumple el dialecto en Bienvenue chez les Ch'tis es su empleo como tema de conversación. En muchas ocasiones se habla explícitamente del dialecto así como de algunas de sus formas, lo que en una traducción al estándar dejaría de tener sentido (función metalingüística). A continuación trataremos las diferentes opciones para traducir el dialecto y las discutiremos a través de ejemplos de la película.

\section{OPCIONES DE EQUIVALENCIA INDIRECTA}

\section{I Verbalización directa}

Si del uso del dialecto se deduce una información puramente objetiva, esta también puede tematizarse de forma explícita —al menos si el montaje no requiere la absoluta sincronización de labios. Un ejemplo de la tematización directa de la procedencia lo encontramos ya en la versión original: «Vous devez aimer l'lavande vu que vous êtes du shud» («Le gusta la lavanda siendo del sur»). Este «vu que vous êtes de....» («siendo de...») también podría utilizarse, en teoría, en otros lugares como un suplemento a la traducción literal. Claro que inserciones de este tipo producen a menudo un efecto artificial, frenan el progreso de la acción y, en la mayoría de los casos, no son posibles debido a la corta duración de algunas intervenciones.

\subsection{Particularidades paralingüisticas}

\subsection{Cualidad de la voz}

Ya se sabe que las características de la voz influyen en la valoración del hablante. Así, en Québec, por ejemplo, se establecen valoraciones diferentes del francés comparado con el inglés, dependiendo de si el hablante es hombre o mujer (cf. Laur 2009). Este hecho puede ser utilizado para el doblaje, en tanto que los rasgos característicos vinculados con el dialecto podrían ser reproducidos mediante una 
determinada cualidad de la voz. Por ejemplo, la connotación de confianza podría transmitirse mediante la elección de un actor de doblaje con una voz grave y sonora, y la connotación de mente sencilla a través de un hablante con una voz ronca.

Si examinamos nuestra película en versión original, llama la atención el contraste entre la voz grave de Philippe y la voz aguda de Antoine y de sus compañeros, que refleja muy bien la oposición entre las facultades de Philippe y la supuesta simpleza del resto. Este contraste, naturalmente, no solo se debe a la diferencia entre norte y sur, sino también a la relación jerárquica entre el jefe y sus empleados. En la versión doblada tenemos el ejemplo de un cambio de voz muy llamativo en el caso del señor Vasseur, el personaje que se distingue en el texto francés por la mayor densidad de picardismos en su lenguaje. Pero, en general, se mantiene el tipo de voz de la versión original. Esto garantiza que se conserven también otras connotaciones ligadas a la cualidad de la voz, que se perderían mediante una compensación del dialecto con un cambio del timbre.

\subsubsection{Maneras de hablar}

$\mathrm{Al}$ igual que la cualidad de la voz, son también interpretados como marcas de la personalidad las maneras de hablar: la intensidad de la voz, la velocidad al hablar y la claridad en la pronunciación.

En cuanto a los habitantes del norte, sería lógico expresar su supuesta inteligencia limitada mediante un modo de hablar lento y con muchas elisiones. Sin embargo, precisamente un modo de hablar llamativamente lento no conviene al avance rápido de la acción de la película y, sobre todo, los frecuentes primeros planos de los personajes hacen necesaria la sincronización de labios.
A eso hay que añadir que ya en la versión original Antoine - de acuerdo con su posición social- vocaliza de forma menos clara que Philippe y que en la película se caracteriza a todos los habitantes ch'tis con un habla gutural. Por eso, se le explica a Philippe en el ejemplo n. ${ }^{\circ}$ I que para hablar bien el picardo hay que añadir a cada frase un hein francés, o sea, un $e b$ español, que tiene que proceder necesariamente de la parte posterior de la garganta.

También, más adelante se vuelve a llamar expresamente la atención de Philippe sobre la necesidad de una pronunciación gutural. Así, el verdadero picardo no llamaría al camarero simplemente garchon 'garçon' (en el doblaje shaval 'chaval'), sino garchooon, o sea, shavaaal (ejemplo n. ${ }^{\circ}$ ):

De esta forma, se aprecia claramente que la manera de hablar ya es una característica constitutiva del original que tiene que mantenerse necesariamente y que, por tanto, no puede además servir para compensar otra característica del original. La única opción aceptable en este campo es la exageración de algunas características, lo que ocurre de nuevo en el caso del señor Vasseur.

\subsection{Variedades de la lengua meta}

Otra opción para la sustitución del picardo es el empleo de un dialecto o una variedad sociolingüística de la lengua meta. Para poder valorar la posible equivalencia de las variedades de distintos idiomas hay que tener en cuenta el correspondiente grado de utilización de sus sistemas de variedades. Examinemos este fenómeno en los idiomas relevantes para Bienvenidos al Norte, el francés y el español (gráfico I).

El gráfico ilustra que la cantidad de variedades diatópicas es similar en ambas lenguas. En los alrededores de París y de Madrid, el lenguaje coloquial es diatópicamente casi neutral. Al ale- 


\section{EJEMPLO I}

Antoine Non, vois ce que, c'est pas compliqué d'parler le ch'timi, par exemple, nous-autres, on dit 156 pas: Pardonnez-moi, je n'ai pas bien saisi le sens de votre question, on dit: Hein?

Hablar el chetimí no esh tan complicado, por ejemplo, noshotrosh no decimos: Perdone, no he captado el sentido de su pregunta, shino: ¿Ehhhh? ¿Eiiii?

\begin{tabular}{ll}
\hline PHILIPPE & Hein? \\
\hline YaNN & Non, non, cha ch'est le un de un, deux, tro-
\end{tabular}
che.

No, esho esh lo que hacen los corderosh, ijaja!

antoine Ouais, parce que il faut que cha chorte eud Es que tiene que shalir de aquí: ¿ehhh? là, là. Heiiin?

\begin{tabular}{lll}
\hline PHILIPPE & Hein? & ¿Ehhh? \\
\hline ANtoine & $\begin{array}{l}\text { Formidable. Au début, quand on commence } \\
\text { à parler le ch'ti ou le picard, on est cousin } \\
\text { avec le picard, faut juste rajouter le hein à la } \\
\text { fin de chaque phrase. Allez-y, essayez un peu } \\
\text { pour vir! }\end{array}$ & $\begin{array}{l}\text { ¡Fenomenal! Al principio cuando se apren- } \\
\text { de el chetimi o el picard, que shon casi } \\
\text { iguales, basta con añadir eh al final de cada } \\
\text { frashe. Venga, ipruebe a ver qué tal! }\end{array}$ \\
\hline PHILIPPE & J'ai compris, hein! & Ya lo pillo, ¡ehhh! \\
\hline ANtoine & Impeccable. & Impecable. \\
\hline PHILIPPE & Ah, d'accord, hein! & De acuerdo, ¡ehhh! \\
\hline ANNABelle & Ça y'est. Vous parlez le ch'timi! & Ya eshtá. Ya habla chetimi. \\
\hline
\end{tabular}

\section{EJEMPLO 2}

\begin{tabular}{lll}
\hline PHILIPPE & Bon. Garchon! & Bien. ¡Shaval! \\
\hline ANtoine & $\begin{array}{l}\text { Non, non. Il faut que cha sorte eud là, là: } \\
\text { Garchooon! }\end{array}$ & No. No tiene que salir de aquí: ¡Shavaaal! \\
\hline PHILIPPE & Garchooon! & ¡Shavaaal! \\
\hline Todos & Voilà! Génial! & Jiji, ¡esho esh! \\
\hline
\end{tabular}

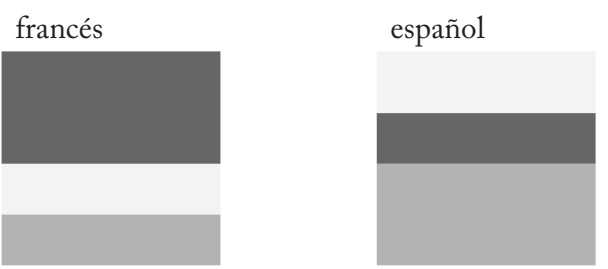

Gráfico I: Existencia de variedades en las diferentes dimensiones de la variación lingüistica en francés y en español (Koch/ Oesterreicher 1990: 235). 
jarse de estos centros, se encuentran formas del francés regional (français regional) más o menos marcadas en Francia (sobre todo, en Alsacia, Córcega, la zona meridional y la picarda, que es la que aquí interesa), en Valonia (Bélgica) y en Romandía (Suiza), así como dialectos secundarios del francés en Canadá, que en las últimas décadas han ganado en importancia gracias a los esfuerzos de normalización. De igual manera, localizamos varios dialectos terciarios (español regional) en el norte de España, en particular, en el País Vasco, Galicia y Cataluña, y llamativos dialectos secundarios en el sur de España, en las Islas Canarias y en Hispanoamérica, para los que entretanto también se han establecido algunas normas propias.

Dentro de la dimensión diastrática encontramos en ambas lenguas jergas con particularidades léxicas. Además, el español conoce muchos rasgos diastráticamente marcados en la fonética y en la morfosintaxis, mientras que el francés apenas incluye marcas diastráticas en su fonética. La dimensión diafásica, por el contrario, contiene numerosas variedades en ambos idiomas. En la dimensión oral podemos distinguir en ambas lenguas características particulares de la lengua hablada. Sin embargo, estas se diferencian considerablemente en cada idioma: mientras que las diferencias entre français parlé y français écrit saltan a la vista, el español casi no muestra formas que pertenezcan exclusivamente al español hablado (cf. Koch/Oesterreicher I990: I42-165, 209-238).

\subsection{Variedad diatópica}

Acabamos de ver que existen divergencias en la cantidad de variedades en las dimensiones orales y diastráticas, pero que el grado de variación en la dimensión determinante para la traducción del picardo fílmico, la dimensión diatópica, es similar en ambas lenguas. Frente a las evidentes diferencias que surgen al comparar el francés con el italiano o con el alemán (cf. Reutner 20Ira/b), esta similitud entre los dialectos franceses y españoles hace considerar como adecuada una posible translación del picardo por un dialecto español.

Aún así, se plantea la pregunta de mediante qué dialecto podría traducirse el picardo de forma adecuada. ¿Debería ser un dialecto de una región que se pueda comparar de alguna manera con la Picardía? ¿Un dialecto que conlleve similares connotaciones a las del picardo? $\mathrm{O}$, más bien, ¿un dialecto que suene de manera similar a este?

En cuanto al estereotipo del clima inhóspito, desde el punto de vista de un español del sur, habría que pensar en el norte de España y utilizar un dialecto norteño. Si, en cambio, se tiene en cuenta la supuesta mente sencilla, se pensaría probablemente en los tópicos a los que se recurre con frecuencia en los medios audiovisuales con respecto a Andalucía, y utilizar, por lo tanto, un dialecto sureño. Pero, aunque autores como Zaro argumentan con la teoría del scopo y consideran esta opción adecuada en algunos casos (200r: 59s.), por lo menos en el nuestro sería una manera de perpetuar y consolidar indeseadamente estereotipos que podrían ofender (cf. los argumentos para una «made-up variety [not] ascribable to any actual Catalan dialect or region» avanzados en Zabalbeascoa 20I2a: 7is.). Además, jandaluz en la Picardía? Se volvería una película extraña y se apartaría mucho del original francés. ¿Tal vez un español del norte de visita en el sur? $\mathrm{O}$, ¿un vallisoletano que sueña con Marbella y que es trasladado a un pueblo gallego? Pero estas serían propuestas para adaptaciones, como la ya realizada en Italia con Benvenuti al Sud, y no representan opciones para nuestro propósito.

En la versión doblada, muchos picardismos 
EJEMPLO 3

Antorne J'vous ai fait signe d'arrêter vot'carète.

Le hice sheñas pa' parar el carro pero no me vio.

Mais vous n'avez vu rin, rin, rin

EJEMPLO 4

\begin{tabular}{lll} 
SR. vasseur & Ahhh, chuis fort content d'vir un ch'ti qui & Bueno, he pensha'o ashercarme a conosher al \\
va s'occuper de min compte en banque. & que va a encargarshe de mi cuenta, porque a \\
Parce qu'il faut pas me raconter des carabis- & mí no me gushta que me cuenten milongas, \\
toules, hein! & ¿shabe ushted? \\
\hline
\end{tabular}

EJEMPLO 5

Tío Ils font des o à la place des $a$. Des que à la place des che. Et les che, ils les font, ils les font... Ils le font, mais à la place des ce.

Dicen $o$ en lugar de $a$. Y she en lugar de ese. Las eses las dicen, las dicen, las dicen, pero en vez de $c e$. (interpretados detalladamente en Reutner 20Ira) son traducidos por expresiones marcadas regionalmente, como en el ejemplo n. ${ }^{\circ} 3$, donde la palabra pic. carète 'coche' se traduce por es. carro, palabra utilizada en algunos países hispanoamericanos para denominar el coche.

Sin embargo, ningún dialecto en particular ha sido empleado de manera constante, lo que también es apropiado, debido a la carencia de una absoluta similitud entre dos regiones, las connotaciones de sus dialectos y su sonido.

\subsubsection{Otras variedades}

Al querer transmitir las connotaciones del dialecto sin evocar una región concreta, se brinda la posibilidad de utilizar variedades diastráticas y, sobre todo, variedades de la dimensión diafásica, que tiene una importancia similar en ambas lenguas. En algunas ocasiones de la versión española de la película, se intenta respetar la marca dialectal de una expresión del texto origen, al emplear un equivalente marcado en el nivel sociolectal. De este modo, en el ejemplo n. ${ }^{\circ}$, pic. carabistoules 'mentiras, cuentos' es traducido por es. milongas (coloq; DRAE, s.v.).

Esta opción del escape hacia una variedad diastrática o diafásica es, sin duda, preferible al uso del léxico no marcado. Sin embargo, hay que reconocer que la equivalencia de las connotaciones del dialecto picardo es insuficiente.

\subsection{La creación de un español artificial}

Tras haber excluido las estrategias anteriores, como última opción queda la sustitución del dialecto por un español artificial creado con esta finalidad. En nuestro caso consiste, sobre todo, en la imitación de cualidades fonológicas del texto origen.

\subsection{Fonología}

El español artificial sigue las características fonológicas del picardo de la película, con las que ya se familiariza al espectador antes de que Philippe llegue al norte, cuando, por ejemplo, el tío de Julie, que parece conocer las realidades de allí, le cuenta a Philippe (ejemplo n. ${ }^{\circ}$ ). 


\begin{tabular}{lll}
\hline Antoine & $\begin{array}{l}\text { Ch'est cha! Hein, c'est comme, euh, ce, ça } \\
\text { devient che et che, ça devient que }\end{array}$ & Y también las eses son shes y las ches son ques. \\
\hline PHilippe & $\begin{array}{l}\text { Ah oui! Par exemple, et oui, c'est les chiens, } \\
\text { c'est des kiens. }\end{array}$ & Y por ejemplo, así, los suyos es los shuyos. \\
\hline ANTOINE & Voilà! & ¡Esho! \\
\hline
\end{tabular}

Cuando Philippe ya tiene más confianza con sus compañeros, a él y al espectador se les explica otra vez (ejemplo n. ${ }^{\circ} 6$ ).

Los últimos dos pasajes contienen las singularidades fonológicas más importantes del picardo de la película, que se diferencia del francés particularmente en el consonantismo por la fricativa [ $\Varangle]$. Aunque este fenómeno no se mantiene sistemáticamente, sí que está presente en toda la versión original. De esta manera, se emplea pic. [ $\Varangle$ ] por fr. [s] y, en consecuencia, pic. [k] por fr. [ $\Varangle]$ —fenómenos documentados, por ejemplo, en pic. canchon en lugar de fr. chanson. Siguiendo de una forma algo libre el modelo de estas peculiaridades fonéticas (y tal vez influenciado por la solución aplicada al mismo fenómeno en auvernés por la versión castellana de Astérix, El escudo arverno), la versión española también recurre especialmente al fonema [ $\Varangle$ ] para sustituir el fonema [s] (así, por ejemplo, encontramos shi por sí, shoy por soy, eshtoy por estoy, esh por es, sheñor por señor, vishto por visto, shuyo por suyo, todosh por todos, sheñas por señas). Además, esta sonorización de la película se refuerza por la frecuente sustitución de la africada [ $\mathrm{f}$ ] por la fricativa [ $\Varangle]$ (así, por ejemplo, se sustituye chaval por shaval, dicho por disho o hecho por hesho). En cambio, el empleo del pic. [k] por fr. [ $\Varangle]$ no encuentra ninguna equivalencia en la versión doblada, mientras que aparece constantemente en los subtítulos optativos, en los que en gran cantidad de ocasiones se han elegido soluciones diferentes, que no se justifican por las necesidades de la subtitulación, sino que documentan preferencias autónomas. En la versión doblada, carente del [k] característico, una adaptación habría sido al menos conveniente cuando Antoine explica en el ejemplo n. ${ }^{\circ}$ 6: «et che, ça deviene que» («y las ches son ques»). No obstante, las características del consonantismo tenidas en cuenta tampoco se llevan a cabo de una manera constante en el doblaje, de manera que con frecuencia se emplea el registro estándar en lugar de la fonética artificial.

E1 vocalismo está aún menos diferenciado del estándar que el consonantismo. La velarización en el picardo, a la que se alude en el ejemplo n. ${ }^{\circ}$, de $a>0$ (por ejemplo, pic. po en vez de fr. pas, pic. sympo en vez de fr. sympa, pic. cho vo en vez de fr. ça va) solo aparece en el doblaje mediante la adopción directa de CocoColo por Coca-Cola. En consecuencia, se tendría que haber modificado la explicación que el tío da sobre el cambio de $a$ por o (cf. no 5), puesto que realmente no se lleva a cabo. También falta una adaptación de otras particularidades del vocalismo en el español artificial, ya que, como hemos visto, solo copia algunos de los rasgos dialectales explicados previamente por el tío. Así, la característica local de la equivalencia de [o] por fr. antiguo [wE], en fr. moderno [wa], 
anNabelle Alors, deux frites fricadelle et un américain, s'il te plaît.

160

\begin{tabular}{lll} 
& cain, s'il te plaît. & por favor. \\
\hline Momo & Martine! Fricadelle et un américain. & ¡Martine! Fricadelle y un americano. \\
\hline ANNABELLE & Quo qu' ch'est que vous voulez? & Y usted, ¿qué quiere? \\
\hline PHiLipPE & Je sais pas, comme vous. & No lo sé, lo mismo. \\
\hline ANNABelLe & Ben... rajoute une frite fricadelle, Momo. & Puesh, pon otra papa fricadelle, por favor. \\
\hline
\end{tabular}

no queda manifiesta en la versión doblada aunque sí que aparezca muchas veces en la película original (por ejemplo, en pic. quo por fr. quoi, pic. avo por fr. avait, pic. éto por fr. était, pic. on voudro por fr. on voudrait). Asimismo, falta una correspondencia de pic. mi y ti por fr. moi y toi, así como una equivalencia de la metátesis del original tanto en el artículo (pic. eul en lugar de fr. le, la) como en los pronombres (pic. eut bouc en lugar de fr. ta bouche).

En general, la imitación fonológica del dialecto en la lengua meta se muestra como un medio eficaz para poder adoptar determinadas explicaciones de la película original. Igualmente, permite la reproducción de malentendidos lingüísticos y, al mismo tiempo, crea un cierto grado de extrañeza sin llegar a que se vuelva incomprensible. Lamentablemente, la solución mimética no se mantiene de forma consecuente a lo largo de toda la película, lo que no solo se opone al propósito de crear extrañeza, sino que también conduce a una contradicción entre las explicaciones teóricas dadas por los personajes y su práctica real.

\subsubsection{Léxico}

Las peculiaridades léxicas del picardo de la película apenas tienen importancia en comparación con las de la fonética. Sin embargo, si un picardismo aparece, una traducción al español estándar naturalmente no es satisfactoria (cf. por ejemplo la traducción de pic. bénache por es. contento, de pic. bieau por es. bonito, de pic. tchiot por es. pequeño). Al menos, la palabra española debería ser adaptada sistemáticamente a la fonética del español artificial (como, por ejemplo, en la traducción de pic. caillèle por es. shilla 'silla', de pic. tchiot por es. shico 'chico', de pic. draps por es. shábanas 'sábanas').

Una mejor opción es, sin duda, el empleo de un extranjerismo, un préstamo o un neologismo con el que se sustituye el regionalismo léxico. Un ejemplo de la utilización de un extranjerismo es la introducción de fr. fricadelle en español (ejemplo n. ${ }^{\circ} 7$ ). En este caso, los estímulos visuales de la película adquieren un papel explicativo, ya que esta especialidad de embutido regional se puede ver en la correspondiente escena en un primer plano, lo que aclara al público español - así como al francés- de qué se trata.

Con es. jvandiús! (ejemplo n.o 8) se adapta gráfica y fonéticamente al español fr. vingt de diousse!, una interjección muy frecuente en la versión original y, por tanto, una característica de la película. Mediante la alusión a Dios, el espectador español puede clasificar fácilmente la expresión como una blasfemia, aunque su origen concreto es igual de comprensible o 
EJEMPLO 8

\begin{tabular}{lll}
\hline ANNABELle & Ça y’est. Vous parlez le ch'timi! & Ya eshtá. ¡Ya habla chetimi! \\
\hline PhilipPe & Oh, putain! & ¡Joder! \\
\hline \multirow{2}{*}{ antoine } & $\begin{array}{l}\text { Ah non! On dit pas putain comme chez } \\
\text { vous. Chez nous, on dit vingt de diousse. }\end{array}$ & $\begin{array}{l}\text { Ah no, no desimos joder como usted. Desi- } \\
\text { mos vandiús. }\end{array}$ \\
\hline PHILIPPE & Vingt de diousse, hein? & Vandiús, ¿eh? \\
\hline
\end{tabular}

\section{EJEMPLO 9}

$\begin{array}{lll}\text { Tío } & \text { C'est des fadas. C'est des fadas. Et quand tu } & \text { Unos tara'os, tara'os! Y cuando crees que lo } \\ \text { crois tout comprendre, tu apprends que ser- } & \text { entiendes todo, descubres que a la fregona la } \\ \text { pillière, ça se dit wassingue. Allooors... } & \text { llaman paliendre. Ufff... }\end{array}$

\section{EJEMPLO IO}

\begin{tabular}{lll}
\hline Hombre & Chalut, Antoine! & ¡Buenash, Antoine! ¿Qué tal? \\
\hline ANtoine & $\begin{array}{l}\text { Cha va, tizaute? Monsieur Abrams, c'est le } \\
\text { nouveau directeur deul poste. }\end{array}$ & $\begin{array}{l}\text { ¿Qué hay, tiote? Señor Abrams, el nuevo } \\
\text { director de correosh. }\end{array}$ \\
\hline PHilippe & Bonjour, Monsieur Tizaute. & Buenos días, Señor Tiote. \\
\hline ANtoine & Bonjour, Monsieur Tizaute? & ¿Buenos días, Señor Tiote? \\
\hline Hомвre & Elle est bien bonne, celle-là! & ¡Ésha shí que esh buena! \\
\hline
\end{tabular}

incomprensible para él que para un espectador francés (cf. Reutner 2012).

Para transmitir la sensación de extrañeza se han creado también expresiones totalmente nuevas. Así, la fregona, denominada en picardo wassingue (ejemplo n. ${ }^{\circ}$ 9), se traduce por paliendre en español, que, si bien suena dialectal, no está documentada en ninguna variedad del español y solo evoca la idea de fregona con el morfema pal(o). La creación del neologismo es necesaria y adecuada para hacer convincente la explicación de la fregona dada en la versión original.

La película también está caracterizada por determinadas formas de tratamiento, lo que la versión española tiene en cuenta, por ejemplo, en la traducción de pic. tizaute. Esta palabra (del pronombre $t i$ 'toi' y autre) se utiliza para un tratamiento amistoso, pero el protagonista no lo entiende en el ejemplo n. ${ }^{\circ}$ Io, cuando se dirige a su interlocutor, al que Antoine ha saludado utilizando tizaute, con la expresión M. Tizaute. El neologismo español tiote mantiene el efecto cómico, garantiza, además, la sincronización de labios por sus dos sílabas y sus dos $t$, al tiempo que evoca la palabra comodín española tío, lo que favorece su compresión.

Otro picardismo muy frecuente en la película es pic. biloute. Su uso se explica en el ejemplo n. ${ }^{\circ}$ II: «tout le monde y s'appelle biloute. [...] ch'est le surnom à tout le monde. [...] Cha veut 
EJEMPLO II

\begin{tabular}{|c|c|c|}
\hline FABRICE & Bravo, biloute! & ¡Bravo, pichula! \\
\hline PHILIPPE & Bravo qui? & Bravo ¿qué? \\
\hline ANTOINE & $\begin{array}{l}\text { Euh, biloute. Euh, tout le monde y s'appelle } \\
\text { biloute. Ici, ch'est, ch'est le surnom à tout le } \\
\text { monde. }\end{array}$ & $\begin{array}{l}\text { Eh, pichula. Todos nos llamamos pichula. } \\
\text { Todos tenemos el mismo mote. }\end{array}$ \\
\hline PHILIPPE & Ça veut dire quoi, biloute? & Y ¿¿qué significa pichula? \\
\hline ANTOINE & $\begin{array}{l}\text { Biloute? Ça veut dire, euh... Cha veut rien } \\
\text { dire, je... }\end{array}$ & Pichula shignifica... no shignifica nada. \\
\hline YANN & Cha veut dire p'tite quéquette. & Shignifica... pichita. \\
\hline PHILIPPE & P'tite quéquette? & ¿Pichita? \\
\hline ANNABELLE & $\begin{array}{l}\text { Oui, enfin, euh, non, non, non, ça n'a rien à } \\
\text { voir avec une quéquette, hein. C'est, ch'est } \\
\text { juste affectueux. }\end{array}$ & $\begin{array}{l}\text { Shí, bueno no... no tiene nada que ver con la } \\
\text { colita, esh más bien cariñoso. }\end{array}$ \\
\hline PHILIPPE & $\begin{array}{l}\text { Aha! D'accord, d'accord! Apprenez-moi des } \\
\text { gros mots justement, c'est important les gros } \\
\text { mots quand on apprend une langue. }\end{array}$ & $\begin{array}{l}\text { Ah, de acuerdo, de acuerdo. Enséñenme } \\
\text { tacos, son esenciales para aprender una len- } \\
\text { gua. }\end{array}$ \\
\hline
\end{tabular}

rien dire. [...] Cha veut dire p'tit quéquette. [...] ça a rien à voir avec une quéquette. [...] ch'est juste affectueux». La versión doblada traduce pic. biloute por es. pichula, una denominación vulgar en Chile y Perú para pene (cf. DRAE, s.v.). «Todos nos llamamos pichula. Todos tenemos el mismo mote. Pichula [...] no significa nada. [...] Significa... pichita. [...] no tiene nada que ver con la colita, esh más bien cariñoso». En el caso de pichula se trata de un neologismo semántico muy acertado que mantiene la importante connotación sexual de la expresión francesa para poder adoptar la explicación que se da en la película al respecto.

\section{CONCLUSIONES}

En resumen, podemos constatar que el dialecto expresa mucho más que la mera procedencia del hablante. En la película Bienvenue chez les Ch'tis se evidencia como elemento central de la acción y de la estética en tres sentidos: en primer lugar, refleja las características atribuidas a los hablantes de ch'ti, como la mente sencilla y la grosería; en segundo lugar, el empleo del dialecto sirve, una y otra vez, como detonante de lo cómico y, en tercer lugar, el dialecto en sí se convierte en tema de conversación en reiteradas ocasiones. Por tanto, para trasmitir las tres funciones del dialecto en español, el traductor tiene que buscar estrategias adecuadas.

$\mathrm{Al}$ analizar las opciones posibles rechazamos la verbalización directa de las connotaciones vinculadas al dialecto, ya que no solo hace demasiado explícitos aspectos subliminales, sino que, además, esta opción es solo posible en ciertas ocasiones debido a la necesaria sincronización de labios. Igual de poco apropiada 
se mostró la sustitución del dialecto mediante características paralingüísticas como la cualidad de la voz o la manera de hablar, puesto que ya realizan otras importantes funciones en el original y, por consiguiente, deberían mantenerse inalteradas en la versión doblada. Además, con ambos procedimientos no es posible trasladar adecuadamente los malentendidos lingüísticos ni las conversaciones sobre el dialecto.

Otra opción que consideramos fue la sustitución del dialecto mediante una variedad de la lengua meta. La comparación de los sistemas de variedades del francés y del español dio como resultado un papel similar de los dialectos en la cultura lingüística de ambos países, lo que podría hacer pensar que la traducción mediante un dialecto español es, en principio, posible. Sin embargo, persiste el obstáculo de no poder comparar dos regiones o dos dialectos desde el punto de vista fonético y connotativo, así como el problema de la deficiencia de autenticidad de un dialecto español hablado en la Picardía.

Dado que la traducción mediante una variedad del español tampoco se presentó como una solución satisfactoria, como última opción queda la creación de un español artificial. En nuestra película, se compone de unos cuantos extranjerismos, préstamos y neologismos en el léxico, pero, sobre todo, se caracteriza por la imitación fonológica de algunas peculiaridades del dialecto de la versión origen. Esta estrategia tiene la ventaja de permitir adoptar las explicaciones que se hacen explícitamente en la película al respecto. $\mathrm{Al}$ mismo tiempo, con este instrumento, también se pueden reproducir los malentendidos lingüísticos y es posible crear la sensación de extrañeza sin correr el riesgo de que la película se vuelva incomprensible para el público. En Bienvenidos al Norte llama la atención que lamentablemente el empleo del español artificial es poco sistemático. No obstante, esta estrategia llevada a cabo consecuentemente parece ser la mejor de las opciones. Por tanto, sugerimos la solución de Bienvenidos al Norte para estudios adicionales que sistematicen sus ventajas y límites según la tradición discursiva del texto origen y profundicen en los diferentes matices que puede adoptar.

RECIBIDO EN OCTUBRE DE 2012

ACEPTADO EN ABRIL DE 2013

VERSIÓN FINAL DE ABRIL DE 2013

\section{REFERENCIAS BIBLIOGRÁFICAS}

\section{Fuentes primarias}

Boon, Dany (2008). Bienvenue chez les Ch'tis. París: Pathé.

Boon, Dany (2009). Bienvenidos al Norte. Barcelona/ Madrid: Cameo.

\section{Fuentes secundarias}

AA.vv. (2004). Il caso Camilleri. Letteratura e storia. Palermo: Sellerio.

Agost, Rosa (1999). Traducción y doblaje: palabras, voces e imágenes. Barcelona: Ariel.

Álvarez, Roman y María Carmen-África Vidal (eds.) (1996). Topics in Translation 8: Translation, Power, Subversión. Clevedon et ál.: Multilingual Matters.

Baker, Mona (1993). «Corpus Linguistics and Translation Studies: Implications and Applications». En Baker et ál., cit., págs. 233-250.

Baker, Mona et ál. (eds.) (1993). Text and Tecbnology: In Honour of John Sinclair. Ámsterdam/Philadelphia: Benjamins.

Bravo, José María (2003). «La investigación en traducción audiovisual en Espana: los textos cinematográficos». En García Peinado/Ortega Arjonilla, cit., págs. 235-252.

Brumme, Jenny y Anna Espunya (eds.) (2012). The Translation of Fictive Dialogue. Ámsterdam/New York: Rodopi.

Chaume, Frederic (2004). Cine y Traducción. Madrid: Cátedra. 
Chaume, Frederic (2012). Audiovisual Translation: Dubbing. Manchester: St. Jerome.

Chesterman, A. et ál. (eds.) (2000). Translation in context. Ámsterdam: Benjamins.

164 Corrius, Montse y Patrick Zabalbeascoa (20II). "Language variation in source texts and their translations. The case of $\mathrm{L}_{3}$ in film translation». Target 23/I, págs. II3-I3O.

Delabastita, Dirk (2002). «A Great Feast of Languages: Shakespeare's Bilingual Comedy in King Henry $V$ and the French Translators». The Translator $8 / 2$, págs. 303-340.

Delabastita, Dirk (2005). «Henry $V$ in the Low Countries: English, Dutch and the Other Languages». En Shakespeare Yearbook 15, págs. 233-250.

Díaz Cintas, Jorge (200I). «Aspectos semióticos en la subtitulación de situaciones cómicas». En Pajares, cit., págs. II9-I30.

Díaz Cintas, Jorge (ed.) (2009). New Trends in Audiovisual Translation. Brístol et ál.: Multilingual Matters.

Donaire, Luisa y Francisco Lafarga (eds.) (I99I). Traducción y adaptación cultural: España-Francia. Oviedo: Universidad de Oviedo.

DRAE: Real Academia Española (2004). Diccionario de la lengua española. Madrid: Espasa.

Duro Moreno, Miguel (ed.) (200I). La traducción para el doblaje y la subtitulación. Madrid: Cátedra.

Fernández, Mercedes y Francoise Gaspin (I99I). «Astérix en español y/o la opacidad de la traducción de un código cultural». En Donaire/Lafarga, cit., págs. 93-IO7.

Franco Aixelá, Javier (I996). «Culture-specific items in Translation». En Álvarez/Vidal, cit., págs. $5^{2}-78$.

Fusari, Sabrina (2007). «Idioletti e dialetti nel doppiaggio italiano de I Simpsons». Quaderni del Centro di Studi Linguistico-Culturali, Bologna, <http :// amsacta.cib.unibo.it/2I82/I/Fusari_OP_COMPLETO.pdf>, págs. 4-35 [consulta 9 de mayo de 20I3].

García Peinado, Miguel A. y Emilio Ortega Arjonilla (eds.) (2003). Panorama actual de la investigación en traducción e interpretación, vol. 2. Granada: Atrio.

Heiss, Christine (2004). «Dubbing Multilingual Films: A New Challenge?». Meta 49/r, págs. 208-220.

Herbst, Thomas (1994). Linguistische Aspekte der Synchronisation von Fernsebserien. Phonetik, Textlinguistik, Übersetzungstheorie (Linguistische
Arbeiten 318). Tubinga: Niemeyer.

Herrero, Leticia (2000). «Sobre la traducibilidad de los marcadores culturales». En Chesterman et ál., cit., págs. 307-316.

Hesse-Quack, Otto (1967).Der Übertragungsprozess bei der Synchronisation von Filmen. Eine interkulturelle Untersuchung. Stuttgart: Cotta.

Kahn, Moshe (2004). «Il dialetto nelle traduzioni di Andrea Camilleri». En AA.vv., cit., págs. I8o-I86.

Koch, Peter / Oesterreicher, Wulf (1990). Gesprochene Sprache in der Romania: Französisch, Italienisch, Spanisch. Tubinga: Niemeyer.

Laur, Elke (2009). "Sprachwahrnehmung in Montreal: Ergebnisse der matched-guise-Methode I960 und 2004». En Reutner, cit., págs. 215-234.

Muñoz Gil, Marta (2009). «Dubbing The Simpsons in Spain: A Case Study». En Díaz Cintas, cit., págs. I42-I57.

Muñiz Muñiz, María de las Nieves (2004). «Lo stile della traduzione: Camilleri in Spagna». En AA.VV., cit., págs. 206-2I2.

Muñoz-Basols, Javier et ál. (2012). The Limits of Literary Translation: Expanding Frontiers in Iberian Languages. Kassel: Reichenberger.

Newmark, Peter (1988). A textbook of translation, Nueva York et ál.: Prentice Hall.

Nida, Eugene [1947] (196I). Bible translating. An Analysis of Principles and Procedures, with Special Reference to Aboriginal Languages. Londres: United Bible Society.

Nord, Christiane (I994). "It's tea-time in Wonderland: culture-markers in fictional texts». En Pürschel, cit., págs. 523-538.

Nord, Christiane (1997). Translating as a Purposeful Activity. Functionalist Approaches Explained. Manchester et ál.: St. Jerome.

Oksaar, Els (I988). Kulturemtheorie. Ein Beitrag zur Sprachverwendungsforschung vorgelegt in der Sitzung vom I7. Januar 1986. Göttingen: Vandenhoeck\&Ruprecht.

Pajares, Eterio et ál. (200I). Trasvases Culturales: Literatura, cine y traducción 2. Zarautz: Universidad del País Vasco.

Pajares Jiménez, María Teresa (20II). «El español 'neutro' y la oralidad». En Sáez, cit., págs.45-58.

Pürschel, Heiner (ed.) (1994). Intercultural Communication. Proceedings of the I7th International L.A.U.D. Symposium. Frankfurt am Main: Lang. 
Ramírez Luengo, José Luis (20II). «Imaginar lo imposible: algunas reflexiones sobre el denominado español neutro». En Daniel Sáez et ál. cit., págs. $17-25$.

Reutner, Ursula (ed.) (2009). 400 Jahre Québec. Kulturkontakte zwischen Konfrontation und Kooperation. Heidelberg: Winter (Studia Romania 153).

Reutner, Ursula (2orra). «Kulturspezifika in der Synchronisation. Zur Kunstsprache in Willkommen bei den Sch'tis». Zeitschrift für Französische Sprache und Literatur I2I/I, págs.I3-38.

Reutner, Ursula (20Irb). «Varietà regionali e doppiaggio cinematografico. La strategia di Giù al Nord». La lingua italiana 7 , págs.Io3-II9.

Reutner, Ursula (2012). "Zur Interjektion vingt de diousse!». Zeitschrift für Romanische Philologie I28/I, págs. I44-I5I.

Reutner, Ursula (en prensa). «La comicidad en el doblaje: cómo un francés del Norte se convierte en un yeti». En Schrader-Kniffki/Jansen, cit.

Roinila, Pauli et ál. (1983). Näkökohtia käänämisen tutkimuksesta. Joensuu: Joensuun korkeakoulu.
Sáez, Daniel et ál. (ed.) (20II). Últimas tendencias en traducción e interpretación. Madrid/Frankfurt am Main: Vervuert/Iberoamericana.

Schrader-Kniffki, Martina y Silke Jansen (eds.) (en prensa). La traducción a través de los tiempos, espacios y disciplinas. Berlín: Franck \& Timme.

Vermeer, Hans (1983). «Translation theory and linguistics». En Roinila et ál. cit., págs. I-IO.

Vittoz, Dominique (2004). «Quale francese per tradurre l'italiano di Camilleri? Una proposta non pacifica». En AA.VV., cit., págs I87-I99.

Zabalbeascoa, Patrick (20I2a). «Translating dialogues in audiovisual fiction». En Brumme/Espunya, cit., págs. $63-78$.

Zabalbeascoa, Patrick (20I2b). «Translating Heterolingual Audiovisual Humor: Beyond the Blinkers of Tradicional Thinking». En Javier MuñozBasols et ál., cit., págs.3I7-338.

Zaro Vera, Juan Jesús (200I). «Conceptos traductológicos para el análisis del doblaje y la subtitulación». En Miguel Duro Moreno, cit., págs. 47-63. 